\title{
Climbing elements in finite Coxeter groups
}

\author{
Thomas Brady \\ School of Mathematical Sciences \\ Dublin City University, Glasnevin, Dublin 9, Ireland \\ tom.brady@dcu.ie \\ Aisling Kenny \\ School of Mathematical Sciences \\ Dublin City University, Glasnevin, Dublin 9, Ireland \\ aisling.kenny9@mail.dcu.ie \\ Colum Watt \\ School of Mathematical Sciences \\ Dublin Institute of Technology, Dublin 8, Ireland \\ colum.watt@dit.ie
}

Submitted: May 9, 2010; Accepted: Nov 8, 2010; Published: Nov 19, 2010

Mathematics Subject Classifications: 20F55, 05E15

\begin{abstract}
We define the notion of a climbing element in a finite real reflection group relative to a total order on the reflection set and we characterise these elements in the case where the total order arises from a bipartite Coxeter element.
\end{abstract}

\section{Introduction}

Suppose $(W, S)$ is a finite Coxeter system. Each reduced expression for an element $w$ of $W$ determines a total order on the inversion set of $w$. The inversion set of the longest element $w_{0}$ of $W$ is equal to the set, $T$, of all the reflections and a particular reduced expression for $w_{0}$ gives a total order, $\leqslant_{T}$, on $T$. For some elements $w$ of $W$, the restriction of $\leqslant_{T}$ to the inversion set of $w$ coincides with the order determined by one of its reduced expressions. We will call such an element $w$ a climbing element of $W$. Geometrically, this means that there is a gallery from the fundamental domain $C$ to $w(C)$ which crosses hyperplanes in increasing order.

In this paper, we characterise the climbing elements in the case where the reduced expression for $w_{0}$ is obtained by iterating a bipartite factorisation of a Coxeter element. 
This characterisation is obtained using the construction from [6] of a copy of the type- $W$ generalised associahedron, whose cone is a coarsening of the fan determined by the $W$ reflection hyperplanes. This coarsening determines an equivalence relation on $W$ whose equivalence classes we prove directly to be intervals in the left weak order. The least elements of these intervals are precisely the climbing elements. It follows that the number of climbing elements is equal to the $W$-Catalan number. The maximal elements in these intervals are translates of the falling elements of $W$, a notion that is analagous to that of climbing elements but which is defined using the reverse of the order $\leqslant_{T}$.

For any minimal factorisation of a Coxeter element, the interval property of the equivalence classes can be deduced from [9] and [10], where the minimal elements are the corresponding Coxeter-sortable elements. Thus we provide a different characterisation of Coxeter-sortable elements in the case of a bipartite factorisation of the Coxeter element. Indeed, the notion of climbing element arose from our attempts to show that the equivalence classes had the interval property without explicitly using Coxeter-sortable elements.

The paper is organised as follows. In $\S 2$ we collect some facts about inversion sets, extend a theorem of Papi and recall some results from [5] and [6] about orderings of roots and the geometry of the generalised associahedron. In $\S 3$ we define climbing elements and we show that each facet of the generalised associahedron determines such an element. We characterise climbing elements in $\S 4$ while in $\S 5$ we introduce and characterise falling elements.

\section{Preliminaries}

\subsection{Inversion sets}

For background on reflection groups, root systems and inversion sets we refer to [3] and [4]. Throughout this paper, $(W, S)$ is a Coxeter system with $W$ finite, acting effectively on $\mathbb{R}^{n}$ and with standard generating set $S=\left\{s_{1}, \ldots s_{n}\right\}$. Denote by $T$ the reflection set of $W$, that is, the set of congugates of elements of $S$. Let $C$ be the fundamental chamber with inward unit normals given by the simple roots $\left\{\alpha_{1}, \ldots, \alpha_{n}\right\}$, where $s_{i}$ is the reflection in the hyperplane normal to $\alpha_{i}$. Let $\left\{\beta_{1}, \ldots, \beta_{n}\right\}$ be the dual basis so that $\alpha_{i} \cdot \beta_{j}=\delta_{i j}$.

For each $w \in W$ we $\operatorname{define}_{\operatorname{Inv}_{\rho}}(w)$ to be the set of positive roots $\lambda$ such that $w^{-1}(\lambda)$ is a negative root. Thus $\operatorname{Inv}_{\rho}(w)$ is the set of positive roots whose orthogonal hyperplanes separate the fundamental chamber $C$ from its image $w(C)$. The corresponding set of reflections is denoted by $\operatorname{Inv}(w)$, that is, $\operatorname{Inv}(w)=\left\{R(\lambda) \mid \lambda \in \operatorname{Inv}_{\rho}(w)\right\}$, where $R(\lambda)$ is the reflection in the hyperplane orthogonal to $\lambda$. We refer to $\operatorname{Inv}(w)$ as the inversion set of $w$. If $w=s_{i_{1}} s_{i_{2}} \ldots s_{i_{k}}$ is a reduced word, then $\operatorname{Inv}(w)=\left\{t_{1}, \ldots, t_{k}\right\}$ where

$$
t_{1}=s_{i_{1}}<t_{2}=s_{i_{1}} s_{i_{2}} s_{i_{1}}<t_{3}=s_{i_{1}} s_{i_{2}} s_{i_{3}} s_{i_{2}} s_{i_{1}}<\ldots
$$

as in section 1.3 of [3]. This defines a linear order on $\operatorname{Inv}(w)$ and the corresponding linear order on $\operatorname{Inv}_{\rho}(w)$ is given by

$$
\alpha_{i_{1}}<s_{i_{1}}\left(\alpha_{i_{2}}\right)<s_{i_{1}} s_{i_{2}}\left(\alpha_{i_{3}}\right)<\ldots
$$


In [8], Papi characterises ordered inversion sets among ordered subsets of $T$. His proof is given for crystallographic groups although he notes that it can be generalised to apply to all Coxeter groups. The following theorem modifies Papi's characterisation and is valid in the general finite case. For completeness the proof is included in an appendix.

Theorem 2.1 An ordered subset $\Sigma$ of positive roots for $W$ is derived from a reduced expression for some element of $W$ if and only if $\Sigma$ satisfies both of the following conditions on triples $\{\sigma, \tau, \rho\}$ of positive roots satisfying $\rho=a \sigma+b \tau$ for some numbers $a>0$ and $b>0$.

(i) Whenever $\sigma$ and $\tau$ are elements of $\Sigma$ with $\sigma<\tau$ then $\rho \in \Sigma$ and $\sigma<\rho<\tau$.

(ii) Whenever $\rho$ is an element of $\Sigma$, then either (a) $\sigma \in \Sigma$ and $\sigma<\rho$ or (b) $\tau \in \Sigma$ and $\tau<\rho$.

\subsection{Geometry of the generalised associahedron}

Let $\Pi^{+}$be the set of all positive roots. We recall from [5] the special features of the linear order on $\Pi^{+}$determined by iterating a so-called bipartite Coxeter element. First assume that the elements of the simple system are ordered so that $\left\{\alpha_{1}, \ldots, \alpha_{s}\right\}$ and $\left\{\alpha_{s+1}, \ldots, \alpha_{n}\right\}$ are orthonormal sets. Let $c=R\left(\alpha_{1}\right) R\left(\alpha_{2}\right) \ldots R\left(\alpha_{n}\right)$ be the corresponding Coxeter element. Because of this partitioning, such a $c$ is called bipartite. If $h$ denotes the order of $c$ then $W$ contains $n h / 2$ reflections. Denoting by $w_{0}$ the longest element of $W$ it follows from the proof of Corollary 4.5 of [11] that $w_{0}$ has the reduced expression

$$
w_{0}=\left\{\begin{array}{cc}
c^{h / 2} & \text { if } h \text { is even } \\
c^{(h-1) / 2} R\left(\alpha_{1}\right) \ldots R\left(\alpha_{s}\right) & \text { if } h \text { is odd }
\end{array}\right.
$$

It follows that the ordered set $\operatorname{Inv}_{\rho}\left(w_{0}\right)$ is equal to $\left\{\rho_{1}, \rho_{2}, \ldots, \rho_{n h / 2}\right\}$ where

$$
\rho_{i}=R\left(\alpha_{1}\right) R\left(\alpha_{2}\right) \ldots R\left(\alpha_{i-1}\right) \alpha_{i}
$$

and we define $\alpha_{i}=\alpha_{i-n}$ for $i>n$. In fact, $\operatorname{Inv}_{\rho}\left(w_{0}\right)=\Pi^{+}$and we denote this order on $\Pi^{+}$by $\leqslant_{\rho}$ and by $\leqslant_{T}$ the corresponding order on the reflection set $T$.

Furthermore, in [5], we define the vectors

$$
\mu_{i}=R\left(\alpha_{1}\right) R\left(\alpha_{2}\right) \ldots R\left(\alpha_{i-1}\right) \beta_{i}, \quad i=1,2, \ldots, n h
$$

where we similarly define $\beta_{i}=\beta_{i-n}$ for $i>n$. It is immediate from the definitions of $\rho_{i}$ and $\mu_{j}$ that $\rho_{i+n}=c\left(\rho_{i}\right), \mu_{j+n}=c\left(\mu_{j}\right)$ and $\rho_{i} \cdot \mu_{i}=1$. We recall that $\mu_{i}=\mu\left(\rho_{i}\right)$ where $\mu$ is the linear map defined by $\mu=2(I-c)^{-1}$. In particular, $\rho_{i}=(1 / 2)(I-c) \mu_{i}$. Furthermore, we have

Proposition 2.2 (Proposition 4.6 of [5])

(a) $\mu_{i} \cdot \rho_{j}=-\mu_{j+n} \cdot \rho_{i}$ for all $i$ and $j$.

(b) $\mu_{i} \cdot \rho_{j} \geqslant 0$, for $1 \leqslant i \leqslant j \leqslant n h / 2$.

(c) $\mu_{i+t} \cdot \rho_{i}=0$, for $1 \leqslant t \leqslant n-1$ and for all $i$.

(d) $\mu_{j} \cdot \rho_{i} \leqslant 0$ for $1 \leqslant i<j \leqslant n h / 2$. 
We recall from [6] that a copy, denoted $\mu A X(c)$, of the type- $W$ associahedron has a facet with vertex set $\left\{\mu\left(\tau_{1}\right), \ldots, \mu\left(\tau_{n}\right)\right\}$ whenever both

$$
\rho_{1} \leqslant \tau_{1}<\tau_{2}<\ldots<\tau_{n} \leqslant \rho_{n h / 2+n} \text { and } c=R\left(\tau_{n}\right) \ldots R\left(\tau_{1}\right) .
$$

We also recall that $\mu A X(c)$ determines a particular coarsening of the Coxeter fan, that is, of the fan defined by the $W$ reflection hyperplanes. This coarsening has rays in the directions $\mu_{1}, \ldots, \mu_{n h / 2+n}$ and each maximal cone is of the form cone $(F)$, where $F$ is a facet $\mu A X(c)$ and cone $(F)$ denotes the positive cone on $F$. We define an equivalence relation on $W$ by $w \sim w^{\prime}$ if and only if $w(C)$ and $w^{\prime}(C)$ are contained in the same maximal cone.

Finally, we will use the filtration of $\mu A X(c)$ inherited from the filtration of $X(c)$ used in [5]. For each root $\rho$ we define the subsets $\rho^{+}, \rho^{-}$and $\rho^{\perp}$ by

$$
\begin{aligned}
& \rho^{+}=\{x \in \mathbb{R} \mid x \cdot \rho \geqslant 0\} \\
& \rho^{-}=\{x \in \mathbb{R} \mid x \cdot \rho \leqslant 0\} \\
& \rho^{\perp}=\{x \in \mathbb{R} \mid x \cdot \rho=0\} .
\end{aligned}
$$

For $n \leqslant i \leqslant n h / 2+n$, we define $V_{i}=\left\{\mu_{1}, \ldots, \mu_{i}\right\}, \mu X_{i}$ to be the subcomplex of $\mu A X(c)$ consisting of those simplices with vertices in $V_{i}$ and

$$
\mu Z_{i}=\rho_{i-n+1}^{+} \cap \rho_{i-n+2}^{+} \cap \ldots \cap \rho_{n h / 2}^{+} .
$$

It follows that the closure of $\mu Z_{i} \backslash \mu Z_{i-1}$ is equal to

$$
\rho_{i-n}^{-} \cap \rho_{i-n+1}^{+} \cap \ldots \cap \rho_{n h / 2}^{+} .
$$

We note that $\mu Z_{n}$ and $\mu Z_{n h / 2+n}$ coincide with the fundamental chamber $C$ and with $\mathbb{R}^{n}$ respectively. We also note that Proposition 7.6 of [5] (in the case $\alpha=c$ ) can be extended to show that $\mu Z_{i}$ coincides with both the positive cone on $\mu X_{i}$ and the positive span of $V_{i}$.

\section{Climbing elements}

In this section we define climbing elements and show that each subset of the vertex set of $\mu A X(c)$ determines a climbing element. In the case of the vertex set of a facet we will show that this climbing element is the minimum in the corresponding equivalence class of $(W, \sim)$.

Definition 3.1 An element $w$ of $W$ is climbing (with respect to the reflection order $\leqslant_{T}$ ) if the order on Inv $(w)$ given by $\leqslant_{T}$ coincides with the order determined by one of the reduced expressions for $w$.

Definition 3.2 For each subset $A$ of $V_{n h / 2+n}$ we define the set $N(A)$ of positive roots by

$$
N(A)=\left\{\rho_{i} \mid 1 \leqslant i \leqslant n h / 2 \text { and } \rho_{i} \cdot \mu \leqslant 0 \text { for all } \mu \in A\right\} .
$$


Thus a positive root $\rho$ belongs to $\mathrm{N}(A)$ if and only if $A \subseteq \rho^{-}$.

Example 3.3 If $A=\left\{\mu\left(\rho_{i}\right)\right\}$ then Proposition 2.2 implies that

$$
N(A)=\left\{\rho_{j}: j<i \text { or } \rho_{j} \cdot \mu\left(\rho_{i}\right)=0\right\} .
$$

For a larger set $B, N(B)$ is the intersection of sets of this form.

Proposition 3.4 For each subset $A$ of $V_{n h / 2+n}$ there exists a element $w \in W$ such that the ordered set $\left(N(A), \leqslant_{\rho}\right)$ coincides with the ordered set $\operatorname{Inv}_{\rho}(w)$ for some reduced expression of $w$. In particular, $w$ is climbing.

Proof: We show that $N(A)$ satisfies the criteria (i) and (ii) of Theorem 2.1. First suppose $\rho_{i}, \rho_{j} \in \mathrm{N}(A)$ with $i<j$ and that $a, b>0$ are such that $\rho_{k}=a \rho_{i}+b \rho_{j}$ is a positive root. For each $\mu \in A$ we have

$$
\rho_{k} \cdot \mu=\left(a \rho_{i}+b \rho_{j}\right) \cdot \mu=a\left(\rho_{i} \cdot \mu\right)+b\left(\rho_{j} \cdot \mu\right) \leqslant 0
$$

since $\rho_{i}, \rho_{j} \in N(A)$. Thus, $\rho_{k} \in \mathrm{N}(A)$. As the order $\leqslant_{\rho}$ on $\Pi^{+}$is derived from a particular reduced expression for the longest element $w_{0}$, the 'only if' part of Theorem 2.1 yields $\rho_{i} \leqslant \rho \rho_{k} \leqslant \rho \rho_{j}$ and criterion (i) follows.

Next, suppose that $\rho_{i}$ and $\rho_{j}$ are positive roots with $i<j$ and that $a, b>0$ are such that $\rho_{k}=a \rho_{i}+b \rho_{j} \in \mathrm{N}(A)$. As in the previous paragraph, Theorem 2.1 yields $\rho_{i} \leqslant \rho \rho_{k} \leqslant \rho \rho_{j}$. It remains to show that $\rho_{i} \in \mathrm{N}(A)$.

If $\rho_{i} \notin \mathrm{N}(A)$ then $\rho_{i} \cdot \mu>0$ for some $\mu \in A$. By definition of $\mu A X(c), \mu=\mu\left(\rho_{q}\right)$ for some root $\rho_{q}$ with $1 \leqslant q \leqslant n h / 2+n$. In fact, $1 \leqslant q \leqslant n h / 2$ since $\left\{\mu_{n h / 2+1}, \ldots, \mu_{n h / 2+n}\right\}$ are the rays of the cone $w_{0}(C)$, the opposite chamber to $C$. Now part (d) of Proposition 2.2 gives $q \leqslant i$. Therefore $q<j$ and, hence, part (b) of Proposition 2.2 implies that $\rho_{j} \cdot \mu=$ $\rho_{j} \cdot \mu\left(\rho_{q}\right) \geqslant 0$. Thus

$$
\rho_{k} \cdot \mu=\left(a \rho_{i}+b \rho_{j}\right) \cdot \mu=a\left(\rho_{i} \cdot \mu\right)+b\left(\rho_{j} \cdot \mu\right) \geqslant a\left(\rho_{i} \cdot \mu\right)>0,
$$

contradicting the assumption that $\rho_{k} \in \mathrm{N}(A)$.

If $F$ is a facet of $\mu A X(c)$, we denote its set of vertices by $V_{F}$. That is $V_{F}=F \cap V_{n h / 2+n}$. Such vertex sets will be particularly important in the sequel.

Proposition 3.5 If $F$ is a facet of $\mu A X(c)$ and $x_{F} \in W$ is the climbing element with $\operatorname{Inv}_{\rho}\left(x_{F}\right)=N\left(V_{F}\right)$, then $x_{F}(C) \subseteq \operatorname{cone}(F)$.

Proof: The set cone $(F)$ can be characterised as an intersection of halfspaces determined by the roots $\rho_{i}$. We show that $x_{F}(C)$ is contained in the same intersection. If $\rho_{i}$ is a positive root with $F$ contained in $\rho_{i}^{-}$, then $\rho_{i} \in N\left(V_{F}\right)$. Since $N\left(V_{F}\right)=\operatorname{Inv}_{\rho}\left(x_{F}\right)$, it follows that $x_{F}(C)$ must also be contained in $\rho_{i}^{-}$. On the other hand, if $\rho_{j}$ is a positive root with $F$ contained in $\rho_{j}^{+}$then $\rho_{j} \notin N\left(V_{F}\right)$ since $F$ has nonempty interior and hence cannot be contained in $\rho_{j}^{\perp}$. Thus $x_{F}(C)$ must also be contained in $\rho_{j}^{+}$. 
Corollary 3.6 Each equivalence class of $(W, \sim)$ contains a minimum in the left weak order on $W$.

Proof: Let $F$ be a facet of $\mu A X(c)$ with vertex set $V_{F}$ and let $x_{F}$ be the element of $W$ whose inversion set is $N\left(V_{F}\right)$ (Proposition 3.4). By Proposition 3.5, $x_{F}(C)$ is contained in cone $(F)$. If $w \sim x_{F}$ then $w(C) \subseteq$ cone $(F)$, by definition, and it follows that $w(C) \subset \rho_{i}^{-}$ for each $\rho_{i} \in N\left(V_{F}\right)$. Thus $N\left(V_{F}\right) \subseteq \operatorname{Inv}_{\rho}(w)$ and Proposition 3.1.3 of [3] now implies that $x_{F}$ precedes $w$ in the left weak order on $W$.

\section{Characterising climbing elements}

The proof of Corollary 3.6 shows that the number of facets of $\mu A X(c)$ does not exceed the number of climbing elements. In fact the theorem below implies that these two numbers are equal. The number of facets of $\mu A X(c)$ is one of the quantities counted by the $W$ Catalan number. For a description of these numbers and their properties see Chapter 1 of $[1]$.

Lemma 4.1 If $\mu\left(\rho_{i}\right)$ is the last vertex of a facet $F$ of $\mu A X(c)$ and if $w$ is a climbing element for which $w(C) \subset$ cone $(F)$, then $R\left(\rho_{i-n}\right) w$ is also a climbing element.

Proof: Assume that $\mu\left(\rho_{i_{1}}\right), \mu\left(\rho_{i_{2}}\right), \ldots, \mu\left(\rho_{i_{n-1}}\right), \mu\left(\rho_{i}\right)$ are the vertices of $F$ where $1 \leqslant$ $i_{1}<\ldots<i_{n-1}<i \leqslant n h / 2+n$ and $c=R\left(\rho_{i}\right) R\left(\rho_{i_{n-1}}\right) \ldots R\left(\rho_{i_{1}}\right)$. Since $1 \leqslant i-n \leqslant n h / 2$ and

$$
c=R\left(\rho_{i}\right) R\left(\rho_{i_{n-1}}\right) \ldots R\left(\rho_{i_{1}}\right)=R\left(\rho_{i_{n-1}}\right) \ldots R\left(\rho_{i_{1}}\right) R\left(\rho_{i-n}\right),
$$

Lemma 2.2 of [2] implies that $\rho_{i-n} \cdot \mu\left(\rho_{i_{k}}\right)=0$ for $k=1,2, \ldots, n-1$. Thus the face of $F$ opposite to the vertex $\mu\left(\rho_{i}\right)$ is contained in the hyperplane $\rho_{i-n}^{\perp}$. It follows that

$$
F \subseteq \overline{\mu Z_{i} \backslash \mu Z_{i-1}}=\rho_{i-n}^{-} \cap \rho_{i-n+1}^{+} \cap \ldots \cap \rho_{n h / 2}^{+}
$$

and, hence, the last wall crossed by any increasing gallery for $w$ is $\rho_{i-n}^{\perp}$. If we delete the last chamber from such an increasing gallery, we obtain an increasing gallery for $R\left(\rho_{i-n}\right) w$. Therefore $R\left(\rho_{i-n}\right) w$ is a climbing element, as required.

Theorem 4.2 Each equivalence class of $(W, \sim)$ contains exactly one climbing element. In particular the number of climbing elements is equal to the $W$-Catalan number.

Proof: Fix an associahedron facet $F$ whose vertices are $\mu\left(\rho_{i_{1}}\right), \mu\left(\rho_{i_{2}}\right), \ldots, \mu\left(\rho_{i_{n-1}}\right), \mu\left(\rho_{i}\right)$ where $1 \leqslant i_{1}<\ldots<i_{n-1}<i \leqslant n h / 2+n$ and $c=R\left(\rho_{i}\right) R\left(\rho_{i_{n-1}}\right) \ldots R\left(\rho_{i_{1}}\right)$. We need to show that there is only one climbing element $w \in W$ for which $w(C) \subset \operatorname{cone}(F)$. Our proof is by induction on $i$.

First note that $i \geqslant n$ and if $i=n$ then $\operatorname{cone}(F)$ must coincide with the fundamental domain $C$. In this case the identity element of $W$ is the only element for which $w(C) \subset$ cone $(F)$. 
Assume now that $i>n$ and that for each associahedron facet $F^{\prime} \subseteq \mu Z_{i-1}$ there is a unique climbing element $w^{\prime}$ for which $w^{\prime}(C) \subseteq \operatorname{cone}\left(F^{\prime}\right)$. Let $G$ be the only other associahedron facet which contains the face $F \cap \rho_{i-n}^{\perp}$. Since $\mu\left(\rho_{i}\right) \cdot \rho_{i-n}<0, G$ is contained in $\mu Z_{i-1}$. Then $\left[R\left(\rho_{i-n}\right) w\right](C)$ also lies in cone $(G)$ since $G$ shares the face $\rho_{i-n}^{\perp} \cap F$ with $F$. As $R\left(\rho_{i-n}\right) w$ is climbing (by Lemma 4.1), the induction hypothesis implies that $R\left(\rho_{i-n}\right) w=w^{\prime}$, the unique climbing element for which $w^{\prime}(C) \subseteq \operatorname{cone}(G)$. Hence $w=R\left(\rho_{i-n}\right) w^{\prime}$ is uniqely determined.

Corollary 4.3 The set of climbing elements in $W$ coincides with the set of Coxetersortable elements of $W$.

Proof: By Theorem 1.1 of [9] the Coxeter-sortable elements of $W$ are precisely the minima of the equivalence classes of $(W, \sim)$. By Theorem 4.2 and the proof of Corollary 3.6, the climbing elements are also the minima of these equivalence classes.

\section{$5 \quad$ Falling elements}

In this section we show that each equivalence class of $(W, \sim)$ contains a maximum in the left weak order on $W$. Just as a climbing element is reached from the fundamental chamber $C$ via a gallery which crosses hyperplanes in increasing order, each of these maxima is reached from the opposite chamber $w_{0}(C)$ via a gallery which crosses hyperplanes in decreasing order. In order to use the results of sections 3 and 4 our strategy is to rebuild the fan determined by $\mu A X(c)$ with $w_{0}(C)$ taking the place of $C$ and $c^{-1}$ taking the place of $c$. This will give an ordering on $T$ which is the reverse of the order $\leqslant_{T}$ and we will refer to the corresponding notion of climbing element as a falling element. The required maxima will then have the form $f w_{0}$ where $f$ is falling.

Since the inward pointing normals for $w_{0}(C)$ are just the negatives of the inward pointing normals for $C$, the new simple system will be $\left\{-\alpha_{1}, \ldots,-\alpha_{n}\right\}$. We will order this simple system by using the corresponding order on the dual basis. Sometimes this order is different than the order $-\alpha_{n}, \ldots,-\alpha_{1}$ but we will see that it gives the reverse of the order $\leqslant_{T}$ on $T$.

Definition 5.1 For $1 \leqslant j \leqslant n$ we define $\beta_{i}^{\prime}=\mu_{n h / 2+n-i+1}$ and we define $\left\{\alpha_{1}^{\prime}, \ldots, \alpha_{n}^{\prime}\right\}$ to be the dual basis to $\left\{\beta_{1}^{\prime}, \ldots, \beta_{n}^{\prime}\right\}$.

Proposition 5.2 The set $\left\{\beta_{1}^{\prime}, \ldots, \beta_{n-s}^{\prime}\right\}$ is a permutation of $\left\{-\beta_{s+1}, \ldots,-\beta_{n}\right\}$ and the set $\left\{\beta_{n-s+1}^{\prime}, \ldots, \beta_{n}^{\prime}\right\}$ is a permutation of $\left\{-\beta_{1}, \ldots,-\beta_{s}\right\}$.

Proof: This follows from Steinberg's proof of Theorem 4.2 of [11], where the vectors he denotes by $\sigma$ and $\tau$ lie in the non-negative linear spans of our $\beta_{1}, \ldots, \beta_{s}$ and $\beta_{s+1}, \ldots, \beta_{n}$, respectively.

Corollary 5.3 The set $\left\{\alpha_{1}^{\prime}, \ldots, \alpha_{n-s}^{\prime}\right\}$ is a permutation of $\left\{-\alpha_{s+1}, \ldots,-\alpha_{n}\right\}$ while the set $\left\{\alpha_{n-s+1}^{\prime}, \ldots, \alpha_{n}^{\prime}\right\}$ is a permutation of $\left\{-\alpha_{1}, \ldots,-\alpha_{s}\right\}$. In particular, the product $c^{-1}=$ $R\left(\alpha_{1}^{\prime}\right) R\left(\alpha_{2}^{\prime}\right) \ldots R\left(\alpha_{n}^{\prime}\right)$ is a bipartite factorisation. 
Definition 5.4 With the convention that $\alpha_{i+n}^{\prime}=\alpha_{i}^{\prime}$ and $\beta_{i+n}^{\prime}=\beta_{i}^{\prime}$, we define $\mu_{i}^{\prime}=$ $R\left(\alpha_{1}^{\prime}\right) R\left(\alpha_{2}^{\prime}\right) \ldots R\left(\alpha_{i-1}^{\prime}\right) \beta_{i}^{\prime}$ and $\rho_{i}^{\prime}=R\left(\alpha_{1}^{\prime}\right) R\left(\alpha_{2}^{\prime}\right) \ldots R\left(\alpha_{i-1}^{\prime}\right) \alpha_{i}^{\prime}$.

Note that $\mu_{j}^{\prime}=\beta_{j}^{\prime}$ for $1 \leqslant j \leqslant n$ and $\mu_{i+n}^{\prime}=c^{-1} \mu_{i}^{\prime}$.

Proposition 5.5 The vectors $\mu_{j}^{\prime}$ and $\rho_{i}^{\prime}$ are related to $\mu_{j}$ and $\rho_{i}$ by $\mu_{j}^{\prime}=\mu_{n h / 2+n-j+1}$ and $\rho_{i}^{\prime}=-\rho_{n h / 2-i+1}$, for $1 \leqslant j \leqslant n h / 2+n$ and $1 \leqslant i \leqslant n h / 2$ respectively.

Proof: For the first identity, write $j=m n+k$ with $0 \leqslant k<n$. Then

$$
\begin{aligned}
\mu_{j}^{\prime} & =R\left(\alpha_{1}^{\prime}\right) \ldots R\left(\alpha_{j-1}^{\prime}\right) \beta_{j}^{\prime} \\
& =\left[c^{-1}\right]^{m} R\left(\alpha_{1}^{\prime}\right) \ldots R\left(\alpha_{k-1}^{\prime}\right) \beta_{k}^{\prime} \\
& =c^{-m} \beta_{k}^{\prime} \text { since } \beta_{k}^{\prime} \perp \alpha_{1}^{\prime}, \ldots, \alpha_{k-1}^{\prime} \\
& =c^{-m} \mu_{n h / 2+n-k+1} \\
& =\mu_{n h / 2+n-m n-k+1} \\
& =\mu_{n h / 2+n-j+1} .
\end{aligned}
$$

For the second identity, we use the first identity and the relationships

$$
c \mu_{i}=\mu_{i}-2 \rho_{i}, \quad c^{-1} \mu_{i}^{\prime}=\mu_{i}^{\prime}-2 \rho_{i}^{\prime}
$$

to get

$$
\begin{aligned}
2 \rho_{i}^{\prime} & =\left(I-c^{-1}\right) \mu_{i}^{\prime} \\
& =\left(I-c^{-1}\right) \mu_{n h / 2+n-i+1} \\
& =\left(I-c^{-1}\right) c \mu_{n h / 2-i+1} \\
& =-(I-c) \mu_{n h / 2-i+1} \\
& =-2 \rho_{n h / 2-i+1},
\end{aligned}
$$

for $1 \leqslant i \leqslant n h / 2$.

We now construct a copy of the type- $W$ associahedron using $c^{-1}$ instead of $c$ and $\left\{\mu_{1}^{\prime}, \ldots, \mu_{n h / 2+n}^{\prime}\right\}$ instead of $\left\{\mu_{1}, \ldots, \mu_{n h / 2+n}\right\}$. We find that the geometric complex is exactly the same since the vertex sets coincide by Proposition 5.5 and there is a facet on a set

$$
\left\{\mu_{i_{1}}^{\prime}, \ldots, \mu_{i_{n}}^{\prime}\right\}
$$

if and only if there is facet on the corresponding set

$$
\left\{\mu_{n h / 2+n-i_{1}+1}, \ldots, \mu_{n h / 2+n-i_{n}+1}\right\} .
$$

The reflection ordering $\rho_{1}^{\prime}, \rho_{2}^{\prime}, \rho_{3}^{\prime}, \ldots$ is the reverse of $\leqslant_{T}$ and determines a different notion of climbing element which we will now call falling. 
Definition 5.6 An element $w$ of $W$ is falling if the order on Inv( $w)$ given by the reverse of the total order $\leqslant_{T}$ coincides with the order determined by one of the reduced expressions for $w$.

The results of sections 3 and 4 apply to give

Theorem 5.7 Each equivalence class of $(W, \sim)$ determines a unique falling element $f$. The element $f w_{0}$ is the maximal element in the corresponding equivalence class in the left weak order.

Corollary 5.8 Each equivalence class of $(W, \sim)$ is an interval in the left weak order on $W$.

\section{Appendix}

Before proving Theorem 2.1, we prove some elementary facts.

Lemma 6.1 If the positive root $\rho$ is not simple then we can write $\rho=a \sigma+b \tau$ for some real numbers $a, b>0$ and some positive roots $\sigma, \tau$.

Proof: First, if $\rho$ is any positive root then we can write

$$
\rho=a_{1} \alpha_{1}+\ldots+a_{n} \alpha_{n} \quad \text { with } a_{i} \geqslant 0 \text { for } 1 \leqslant i \leqslant n
$$

and it follows that

$$
0<\rho \cdot \rho=\rho \cdot\left(\sum_{i} a_{i} \alpha_{i}\right)=\sum_{i} a_{i}\left(\rho \cdot \alpha_{i}\right) .
$$

yielding $\rho \cdot \alpha_{i}>0$ for some simple root $\alpha_{i}$.

Now suppose that $\rho$ is a non-simple, positive root and that $\alpha_{i}$ is a simple root with $\rho \cdot \alpha_{i}>0$ as above. Since $\rho$ is not a simple root, it follows that $s_{i}(\rho)=\sigma$ is a positive root. However, $\sigma=\rho-b \alpha_{i}$, and hence $\rho=\sigma+b \alpha_{i}$ where $b=2\left(\rho \cdot \alpha_{i}\right)>0$, as required.

Define the vector $v_{0}$ by $v_{0}=\beta_{1}+\ldots+\beta_{n}$ and note that $v_{0}$ lies in the interior of the fundamental chamber $C$ since $v_{0} \cdot \alpha_{i}=1$, for each $i$. Note also that for each $w \in W$, the set $\operatorname{Inv}_{\rho}(w)$ is equal to the set of positive roots $\lambda$ such that $\lambda \cdot w\left(v_{0}\right)<0$.

Lemma 6.2 If $w \in W$ and $w(\sigma) \in \operatorname{Inv}_{\rho}(w)$ then $\sigma$ is a negative root.

Proof: Directly from the definition of $\operatorname{Inv}_{\rho}(w)$ we have

$$
\sigma \cdot v_{0}=w(\sigma) \cdot w\left(v_{0}\right)<0 .
$$

Proof of Theorem 2.1: First, assume that the ordered set $\Sigma$ is derived from a reduced expression $w=s_{i_{1}} s_{i_{2}} \ldots s_{i_{k}}$ for some element $w \in W$. Extend this to a reduced expression

$$
s_{i_{1}} s_{i_{2}} \ldots s_{i_{n h / 2}}
$$


for the longest element of $W$, as in Section 1.8 of [7]. For each $1 \leqslant j \leqslant n h / 2$, let $w_{j}=s_{i_{1}} s_{i_{2}} \ldots s_{i_{j}}$ be the $j$ th prefix of this expression and note that $w=w_{k}$.

For condition (i), assume that $\sigma<\tau$ are elements of $\Sigma$ and that $\rho=a \sigma+b \tau$ is a positive root for some $a, b>0$. Then $R(\sigma)=t_{r_{1}}, R(\rho)=t_{r_{2}}$ and $R(\tau)=t_{r_{3}}$ for some $1 \leqslant r_{1}<r_{3} \leqslant k$ (by our assumption on $\Sigma$ ) and some $1 \leqslant r_{2} \leqslant n h / 2$, and where the $t_{j}$ are given by equation (1). We show that $r_{1}<r_{2}<r_{3}$ by eliminating the other possibilities. It then follows that $\rho \in \Sigma$. First, if $r_{2}<r_{1}<r_{3}$ then

$$
w_{r_{2}}\left(v_{0}\right) \cdot \sigma>0 \text { and } w_{r_{2}}\left(v_{0}\right) \cdot \tau>0 \text { while } w_{r_{2}}\left(v_{0}\right) \cdot \rho<0 .
$$

This is impossible since $\rho$ is a positive linear combination of $\sigma$ and $\tau$. Similarly, if $r_{1}<$ $r_{3}<r_{2}$ then

$$
w_{r_{3}}\left(v_{0}\right) \cdot \sigma<0 \text { and } w_{r_{3}}\left(v_{0}\right) \cdot \tau<0 \text { while } w_{r_{3}}\left(v_{0}\right) \cdot \rho>0
$$

which is also impossible since $\rho$ is a positive linear combination of $\sigma$ and $\tau$.

For condition (ii), assume that $\sigma$ and $\tau$ are positive roots and that $a, b>0$ are such that $\rho=a \sigma+b \tau$ is an element of $\Sigma$. Thus $R(\rho)=t_{r}$, for some $1 \leqslant r \leqslant k$, and hence $w_{r}\left(v_{0}\right) \cdot \rho<0$. Since $\rho$ is a positive linear combination of $\sigma$ and $\tau$, at least one of $w_{r}\left(v_{0}\right) \cdot \sigma$ and $w_{r}\left(v_{0}\right) \cdot \tau$ must be strictly negative. Thus, either $R(\sigma) \in \operatorname{Inv}\left(w_{r}\right)$ and hence $\sigma \leqslant \rho$ or $R(\tau) \in \operatorname{Inv}\left(w_{r}\right)$ and hence $\tau \leqslant \rho$. As $a, b>0$, we can exclude the possibilities of $\sigma=\rho$ or $\tau=\rho$.

For the converse, assume that $\Sigma$ is a set of positive roots which satisfies conditions (i) and (ii). As in [8], we proceed by induction on the cardinality of $\Sigma$. To start the induction we assume that $\Sigma=\{\rho\}$. It suffices to show that $\rho$ is a simple root, for then $(R(\rho)$ is the required group element. If $\rho$ is not a simple root, then Lemma 6.1 implies that $\rho=a \sigma+b \tau$ for some other positive roots $\sigma$ and $\tau$ and some $a, b>0$. By condition (ii), either $\sigma$ or $\tau$ is also in $\Sigma$, contradicting the assumption that $\Sigma$ has cardinality one.

For the inductive step, assume that $k>1$ and that the result is true for sets of cardinality less than $k$. Assume that $\Sigma=\left\{\rho_{1}, \rho_{2}, \ldots, \rho_{k}\right\}$ satisfies conditions (i) and (ii). Then the ordered set $\Sigma^{\prime}=\left\{\rho_{1}, \rho_{2}, \ldots, \rho_{k-1}\right\}$ also satisfies these two conditions and hence there is a reduced expression $u=s_{i_{1}} s_{i_{2}} \ldots s_{i_{k-1}}$, of some element $u \in W$, such that

$$
\rho_{1}=\alpha_{i_{1}}, \quad \rho_{2}=s_{i_{1}}\left(\alpha_{i_{2}}\right), \quad \ldots, \quad \rho_{k-1}=s_{i_{1}} s_{i_{2}} \ldots s_{i_{k-2}}\left(\alpha_{i_{k-1}}\right) .
$$

If $u^{-1}\left(\rho_{k}\right)$ is a simple root, $\alpha_{i_{k}}$ say, then the positivity of $\alpha_{i_{k}}$ implies that $l\left(s_{i_{1}} s_{i_{2}} \ldots s_{i_{k}}\right)=$ $l(u)+1$ and, hence, $w=u s_{i_{k}}=s_{i_{1}} s_{i_{2}} \ldots s_{i_{k}}$ is the required minimal expression. Thus it remains to show that $u^{-1}\left(\rho_{k}\right)$ must be simple.

Assume that $u^{-1}\left(\rho_{k}\right)$ is not simple. As $\rho_{k} \notin \operatorname{Inv}_{\rho}(u)$, it follows that $u^{-1}\left(\rho_{k}\right)$ is a positive root. Then $u^{-1}\left(\rho_{k}\right)=a \sigma+b \tau$ for some positive roots $\sigma$ and $\tau$ and some real numbers $a, b>0$, by Lemma 6.1. Thus

$$
\rho_{k}=a u(\sigma)+b u(\tau) .
$$

In order to apply condition (ii) to this equation, we need to show that neither $u(\sigma)$ nor $u(\tau)$ can be a negative root. For example, if $u(\sigma)$ is negative, then

$$
-u(\sigma) \cdot v_{0}>0 \text { and } \quad-u(\sigma) \cdot u\left(v_{0}\right)=-\sigma \cdot v_{0}<0
$$


putting $-u(\sigma)$ in $\operatorname{Inv}_{\rho}(u)$. Thus $-u(\sigma)=\rho_{i}$ for some $i<k$. Condition (i) applied to the expression $u(\tau)=(1 / b) \rho_{k}+(a / b) \rho_{i}$ now implies that $u(\tau) \in \Sigma$ and $u(\tau)<\rho_{k}$, so that, in fact, $u(\tau) \in \Sigma^{\prime}=\operatorname{Inv}_{\rho}(u)$. This gives a contradiction by Lemma 6.2.

Thus both $u(\sigma)$ and $u(\tau)$ must be positive and by Lemma 6.2 again neither belong to $\operatorname{Inv}_{\rho}(u)$. This gives a contradiction since condition (ii) applied to equation (2) implies that one of $u(\sigma)$ and $u(\tau)$ is in $\Sigma$ and precedes $\rho_{k}$, putting one of $u(\sigma)$ and $u(\tau)$ in $\Sigma^{\prime}=\operatorname{Inv}_{\rho}(u)$.

\section{References}

[1] D. Armstrong, Generalized Noncrossing Partitions and Combinatorics of Coxeter Groups, Memoirs of AMS, vol. 202, no. 949, AMS, Providence, 2009.

[2] C.A. Athanasiadis, T. Brady, J. McCammond and C. Watt, h-vectors of generalized associahedra and noncrossing partitions, Int. Math. Res. Not. 2006, Art. ID 69705, $28 \mathrm{pp}$

[3] A. Björner and F. Brenti, Combinatorics of Coxeter groups, Graduate Texts in Mathematics, vol. 231, Springer, New York, 2005.

[4] N. Bourbaki, Lie groups and Lie algebras. Chapters 7-9. Translated from the 1975 and 1982 French originals by Andrew Pressley. Elements of Mathematics (Berlin). Springer-Verlag, Berlin, 2005.

[5] T. Brady and C. Watt, Noncrossing Partition Lattices in finite real reflection groups, Trans. Amer. Math. Soc. 360 (2008), 1983-2005.

[6] T. Brady and C. Watt, From Permutahedron to Associahedron, Proc. Edinburgh. Math. Soc. 53 (2010), 299-310.

[7] J.E. Humphreys, Reflection groups and Coxeter groups, Cambridge Studies in Advanced Mathematics 29, Cambridge University Press, Cambridge, England, 1990.

[8] P. Papi, A characterization of a special ordering in a root system, Proc. Amer. Math. Soc. 120 (1994), no. 3, 661-665.

[9] N. Reading, Sortable elements and Cambrian lattices Algebra Universalis 56 (2007), no. 3-4, 411-437.

[10] N. Reading and D. Speyer, Cambrian fans, J. Eur. Math. Soc. (JEMS) 11 (2009), no. 2, 407-447.

[11] R. Steinberg, Finite reflection groups, Trans. Amer. Math. Soc. 91, No. 3, (1959) 493-504. 